\title{
Proof of a Conjecture of Farkas and Kra
}

\author{
Nian Hong Zhou
}

\begin{abstract}
In this paper we prove a conjecture of Farkas and Kra, which is a modular equation involving a half sum of certain modular form of weight 1 for congruence subgroup $\Gamma_{1}(k)$ with any prime $k$. We prove that their conjecture holds for all odd integers $k \geq 3$. A new modular equation of Farkas and Kra type is also established.
\end{abstract}

\section{Introduction and statement of results}

In this paper, we let $z \in \mathbb{C}, \tau \in \mathbb{C}$ with $\Im(\tau)>0$ and $q=e^{2 \pi \mathrm{i} \tau}$. The theta function with characteristic $\left[\begin{array}{c}\epsilon \\ \epsilon^{\prime}\end{array}\right] \in \mathbb{R}^{2}$ is defined by

$$
\theta\left[\begin{array}{c}
\epsilon \\
\epsilon^{\prime}
\end{array}\right](z, \tau)=\sum_{n \in \mathbb{Z}} \exp \left\{2 \pi \mathrm{i}\left(\frac{1}{2}\left(n+\frac{\epsilon}{2}\right)^{2} \tau+\left(n+\frac{\epsilon}{2}\right)\left(z+\frac{\epsilon^{\prime}}{2}\right)\right)\right\}
$$

which is a generalization of the Jacobi theta functions. The theory of above theta function was systematically studied by Farkas and Kra [2, which plays an important role in combinatorial number theory, algebraic geometry and physics.

In [2, Chapter 4], Farkas and Kra treated the theta function (1.1) with $\epsilon, \epsilon^{\prime} \in \mathbb{Q}$ and $z=0$, that is, the theta constants with rational characteristics. Their derived many interesting results, one of them is the following (see [2, Theorem 9.8, p. 318] and [3]):

Theorem 1.1. For each odd prime $k$ and all $\tau \in \mathbb{C}$ with $\Im(\tau)>0$,

$$
\frac{d}{d \tau} \log \left(\frac{\eta(k \tau)}{\eta(\tau)}\right)+\frac{1}{2 \pi \mathrm{i}(k-2)} \sum_{0 \leq \ell \leq(k-3) / 2}\left(\frac{\theta^{\prime}\left[\begin{array}{c}
1 \\
(1+2 \ell) / k
\end{array}\right](0, \tau)}{\theta\left[\begin{array}{c}
1 \\
(1+2 \ell) / k
\end{array}\right](0, \tau)}\right)^{2}
$$

is a cusp 1-form (cusp form of weight 1 ) for the Hecke congruence subgroup $\Gamma_{0}(k)$. This form is identically zero provided $k \leq 19$. Here $\eta(\tau)=q^{1 / 24} \prod_{n \geq 1}\left(1-q^{n}\right)$ is the Dedekind eta function and

$$
\theta^{\prime}\left[\begin{array}{c}
1 \\
(1+2 \ell) / k
\end{array}\right](0, \tau)=\left.\frac{\partial}{\partial z} \theta\left[\begin{array}{c}
1 \\
(1+2 \ell) / k
\end{array}\right](z, \tau)\right|_{z=0}
$$

Received January 3, 2019; Accepted March 10, 2019.

Communicated by Yifan Yang.

2010 Mathematics Subject Classification. Primary: 11F27; Secondary: 11F12, 14K25.

Key words and phrases. theta functions, theta constants, modular equations.

This research was supported by the National Science Foundation of China (Grant No. 11571114). 
They then in [2, Conjecture 9.10, p. 320] (see also [3]) conjectured that $[1.2$ is identically zero for each odd prime $k$ and all $\tau \in \mathbb{C}$ with $\Im(\tau)>0$.

Remark 1.2. We remark that for odd integers $k, \ell$ with $k \geq 3$,

$$
\left[\frac{\partial}{\partial z} \log \left(\theta\left[\begin{array}{c}
1 \\
\ell / k
\end{array}\right](0, \tau)\right)\right]^{2}
$$

is a modular 1-form (modular form of wight 1) for the group:

$$
G(k)=\Gamma_{1}(k):=\left\{\left(\begin{array}{ll}
a & b \\
c & d
\end{array}\right) \in \mathrm{SL}_{2}(\mathbb{Z}):\left(\begin{array}{ll}
a & b \\
c & d
\end{array}\right) \equiv\left(\begin{array}{ll}
1 & * \\
0 & 1
\end{array}\right)(\bmod k)\right\} .
$$

This fact and more related results can be found in [2,3].

The aim of this paper is to give a proof of the conjecture of Farkas and Kra of above. For the simplicity of the proof, we shall introduce the Jacobi theta function $\theta_{2}(z, q)$, which is defined by (see for example [4])

$$
\theta_{2}(z, q)=\sum_{n \in \mathbb{Z}} q^{(2 n+1)^{2} / 8} e^{\mathrm{i}(2 n+1) z}
$$

Hence it is clear that

$$
\theta\left[\begin{array}{l}
1 \\
\epsilon^{\prime}
\end{array}\right](z, \tau)=\theta_{2}\left(\pi z+\frac{\epsilon^{\prime} \pi}{2}, q\right)
$$

and the conjecture of our concern is equivalent to the following.

Conjecture 1.3. For each odd prime $k$ and all $\tau \in \mathbb{C}$ with $\Im(\tau)>0$,

$$
4(k-2) q \frac{d}{d q} \log \left(\frac{\eta(k \tau)}{\eta(\tau)}\right)-\sum_{\substack{0 \leq \ell<k \\ \ell \equiv 1(\bmod 2)}}\left[\frac{\partial}{\partial z} \log \theta_{2}\left(\frac{\ell}{2 k} \pi, q\right)\right]^{2}=0 .
$$

We shall prove a more general result than Conjecture 1.3. To state our main result, we define the following half sum

$$
S_{\delta}(k):=\sum_{\substack{0 \leq \ell<k \\ \ell-k \equiv \delta(\bmod 2)}}\left[\frac{\partial}{\partial z} \log \theta_{2}\left(\frac{\ell}{2 k} \pi, q\right)\right]^{2}
$$

for each integer $k \geq 2$ and each $\delta \in\{0,1\}$. Our main result is the following modular equations.

Theorem 1.4. For all $\tau \in \mathbb{C}$ with $\Im(\tau)>0$, we have if $\delta=0$ then

$$
S_{\delta}(k)=4(k-2) q \frac{d}{d q} \log \left(\frac{\eta(k \tau)}{\eta(\tau)}\right),
$$

and if $\delta=1$ then

$$
S_{\delta}(k)=4 q \frac{d}{d q} \log \left(\frac{\eta(2 k \tau)^{2 k-2}}{\eta(\tau)^{k} \eta(k \tau)^{k-2}}\right) .
$$


We immediately obtain the proof of Conjecture 1.3 by setting $k \in 2 \mathbb{Z}_{+}+1$ and $\delta=0$ in Theorem 1.4 .

Corollary 1.5. Conjecture 1.3 holds for all odd integers $k \geq 3$. In particular, Conjecture 1.3 is true.

We shall give some consequences of Theorem 1.4. For this purpose we first use Lemma 2.2 below to deduce a proposition as follows.

Proposition 1.6. We have

$$
S_{\delta}(k)=\sum_{\substack{0 \leq \ell<k \\ \ell-k \equiv \delta(\bmod 2)}}\left[\tan \left(\frac{\ell \pi}{2 k}\right)-4 \sum_{h=1}^{2 k}(-1)^{h} \sin \left(\frac{\ell h \pi}{k}\right) \sum_{n \geq 1} \frac{q^{h n}}{1-q^{2 k n}}\right]^{2} .
$$

By setting $q=0$ in Theorem 1.4, applying Proposition 1.6 and $(2.3)$ below we obtain the following trigonometric identity, which has been proved in [2,3] by using the theory of modular form.

Corollary 1.7. For each integer $k \geq 2$,

$$
\sum_{\substack{0 \leq \ell<k \\ \ell-k \equiv \delta(\bmod 2)}}\left[\tan \left(\frac{\ell \pi}{2 k}\right)\right]^{2}= \begin{cases}(k-1)(k-2) / 6 & \text { if } \delta=0, \\ k(k-1) / 6 & \text { if } \delta=1 .\end{cases}
$$

From Theorem 1.4. Proposition 1.6 and $(2.3)$, by choosing different pair $(k, \delta)$ one can obtain many Lambert series identities. For example, if we set $(k, \delta)=(3,1)$, then it is easy to see

Corollary 1.8. We have

$$
\left(1+2 \sum_{n \geq 1} \frac{q^{n}+q^{2 n}-q^{4 n}-q^{5 n}}{1-q^{6 n}}\right)^{2}=1+4 \sum_{n \geq 1}\left(\frac{n q^{n}}{1-q^{n}}+\frac{n q^{3 n}}{1-q^{3 n}}-\frac{8 n q^{6 n}}{1-q^{6 n}}\right) .
$$

Our proof of the main theorem is based on the series expansion $(1.3)$ of $\theta_{2}(z, q)$ and the Jacobi triple product identity. The rest of this paper is organized as follows. In the next section, we first establish some primary results for $\theta_{2}(z, q)$. In Section 3, we prove Theorem 1.4.

\section{Primaries}

We shall need the following primary results, which will be used to prove main results of this paper. 
Proposition 2.1. We have

$$
\left(\frac{\partial}{\partial z} \log \theta_{2}(z, q)\right)^{2}=\mathrm{T}_{z, q}\left(\log \theta_{2}(z, q)\right),
$$

where and throughout, $\mathrm{T}_{z, q}$ is a linear operator defined as

$$
\mathrm{T}_{z, q}=-8 q \frac{\partial}{\partial q}-\frac{\partial^{2}}{\partial z^{2}}
$$

Proof. By (1.3) it is clear that

$$
\left(8 q \frac{\partial}{\partial q}+\frac{\partial^{2}}{\partial z^{2}}\right) \theta_{2}(z, q)=0
$$

which means that

$$
\frac{1}{\theta_{2}(z, q)} \frac{\partial^{2}}{\partial z^{2}} \theta_{2}(z, q)=-8 q \frac{\partial}{\partial q} \log \theta_{2}(z, q) .
$$

Then from the basic fact that

$$
\frac{\partial^{2}}{\partial z^{2}} \log \theta_{2}(z, q)=\frac{1}{\theta_{2}(z, q)} \frac{\partial^{2}}{\partial z^{2}} \theta_{2}(z, q)-\left(\frac{\partial}{\partial z} \log \theta_{2}(z, q)\right)^{2}
$$

we complete the proof of the proposition.

We need the Jacobi triple product identity for $\theta_{2}(z, q)$ (see for example [1, 4] ),

$$
\theta_{2}(z, q)=q^{1 / 8} e^{-\mathrm{i} z} \prod_{n \geq 1}\left(1-q^{n}\right)\left(1+e^{-2 \mathrm{i} z} q^{n}\right)\left(1+e^{2 \mathrm{i} z} q^{n-1}\right)
$$

Lemma 2.2. For each $\ell, k \in \mathbb{Z}$ with $\ell \neq k$ and $k>0$,

$$
-\frac{\partial}{\partial z} \log \theta_{2}\left(\frac{\ell}{2 k} \pi, q\right)=\tan \left(\frac{\ell \pi}{2 k}\right)-4 \sum_{h=1}^{2 k}(-1)^{h} \sin \left(\frac{\ell h \pi}{k}\right) \sum_{n \geq 1} \frac{q^{h n}}{1-q^{2 k n}} .
$$

Proof. Taking the logarithmic derivative of $\theta_{2}(z, q)$ respect to $z$ by (2.1), we have the well known Fourier expansion

$$
\frac{\partial}{\partial z} \log \theta_{2}(z, q)=-\tan (z)+4 \sum_{n \geq 1} \frac{(-1)^{n} q^{n}}{1-q^{n}} \sin (2 n z) .
$$

Noticing that

$$
\begin{aligned}
\sum_{n \geq 1} \frac{(-1)^{n} q^{n}}{1-q^{n}} \sin \left(2 n \frac{\ell \pi}{2 k}\right) & =\sum_{h=1}^{2 k} \sum_{n \geq 0} \frac{(-1)^{h} q^{2 n k+h}}{1-q^{2 n k+h}} \sin \left(\frac{\ell h \pi}{k}\right) \\
& =\sum_{h=1}^{2 k}(-1)^{h} \sin \left(\frac{\ell h \pi}{k}\right) \sum_{n \geq 0} \sum_{\ell \geq 1} q^{(2 n k+h) \ell}
\end{aligned}
$$


and 2.2 we immediately obtain that

$$
\frac{\partial}{\partial z} \log \theta_{2}\left(\frac{\ell}{2 k} \pi, q\right)=-\tan \left(\frac{\ell \pi}{2 k}\right)+4 \sum_{h=1}^{2 k}(-1)^{h} \sin \left(\frac{\ell h \pi}{k}\right) \sum_{n \geq 1} \frac{q^{h n}}{1-q^{2 k n}} .
$$

This completes the proof of the lemma.

The following lemma will be used to prove Theorem 1.4 in the next section.

Lemma 2.3. For each $k \in \mathbb{Z}_{+}$,

$$
\left.\mathrm{T}_{z, q}\left(\log \theta_{2}\left(k z, q^{k}\right)\right)\right|_{z=0}=8(k-1) q \frac{d}{d q} \log \left(\frac{\eta(2 k \tau)^{2}}{\eta(k \tau)}\right)
$$

and

$$
\left.\mathrm{T}_{z, q}\left(\log \left(\frac{\theta_{2}\left(k z-\pi / 2, q^{k}\right)}{\theta_{2}(z-\pi / 2, q)}\right)\right)\right|_{z=0}=8 q \frac{d}{d q} \log \left(\eta(k \tau)^{k-3} \eta(\tau)^{2}\right) .
$$

Proof. By 2.2 we have

$$
\frac{\partial^{2}}{\partial z^{2}} \log \theta_{2}(z, q)=-\tan ^{2}(z)-1+8 \sum_{n \geq 1} \frac{(-1)^{n} n q^{n}}{1-q^{n}} \cos (2 n z)
$$

and

$$
\frac{\partial^{2}}{\partial z^{2}} \log \theta_{2}(z-\pi / 2, q)=-\cot ^{2}(z)-1+8 \sum_{n \geq 1} \frac{n q^{n}}{1-q^{n}} \cos (2 n z) .
$$

Hence we obtain that

$$
\begin{aligned}
\left.\frac{\partial^{2}}{\partial z^{2}} \log \theta_{2}(z, q)\right|_{z=0} & =-1+8 \sum_{n \geq 1} \frac{(-1)^{n} n q^{n}}{1-q^{n}} \\
& =-1+16 \sum_{n \geq 1} \frac{2 n q^{2 n}}{1-q^{2 n}}-8 \sum_{n \geq 1} \frac{n q^{n}}{1-q^{n}}
\end{aligned}
$$

and

$$
\begin{aligned}
& \left.\frac{\partial^{2}}{\partial z^{2}} \log \left(\frac{\theta_{2}\left(k z-\pi / 2, q^{k}\right)}{\theta_{2}(z-\pi / 2, q)}\right)\right|_{z=0} \\
= & \lim _{z \rightarrow 0}\left(\cot ^{2}(z)+1-k^{2}\left(\cot ^{2}(k z)+1\right)\right)+8 \sum_{n \geq 1}\left(\frac{k^{2} n q^{k n}}{1-q^{k n}}-\frac{n q^{n}}{1-q^{n}}\right) \\
= & \frac{1-k^{2}}{3}+8 k^{2} \sum_{n \geq 1} \frac{n q^{k n}}{1-q^{k n}}-8 \sum_{n \geq 1} \frac{n q^{n}}{1-q^{n}} .
\end{aligned}
$$

Using the fact that

$$
q \frac{d}{d q} \log \eta(\alpha \tau)=\frac{\alpha}{24}-\sum_{n \geq 1} \frac{\alpha n q^{\alpha n}}{1-q^{\alpha n}}, \quad \alpha \in \mathbb{R}_{+},
$$


and the above we obtain

$$
\left.\frac{\partial^{2}}{\partial z^{2}} \log \theta_{2}(z, q)\right|_{z=0}=8 q \frac{d}{d q} \log \left(\frac{\eta(\tau)}{\eta(2 \tau)^{2}}\right)
$$

and

$$
\left.\frac{\partial^{2}}{\partial z^{2}} \log \left(\frac{\theta_{2}\left(k z-\pi / 2, q^{k}\right)}{\theta_{2}(z-\pi / 2, q)}\right)\right|_{z=0}=8 q \frac{d}{d q} \log \left(\frac{\eta(\tau)}{\eta(k \tau)^{k}}\right) .
$$

Moreover, by (2.1) and the definition of $\eta(\tau)$, it is easy to see that

$$
\theta_{2}(0, q)=2 \frac{\eta(2 \tau)^{2}}{\eta(\tau)}
$$

and

$$
\lim _{z \rightarrow 0} \frac{\theta_{2}(z-\pi / 2, q)}{z}=2 \eta(\tau)^{3} .
$$

Thus for integer $k \geq 1$, application of (2.4) and (2.6) imply that

$$
\begin{aligned}
\left.\mathrm{T}_{z, q}\left(\log \theta_{2}\left(k z, q^{k}\right)\right)\right|_{z=0} & =-8 q \frac{d}{d q} \log \theta_{2}\left(0, q^{k}\right)-\left.\frac{\partial^{2}}{\partial z^{2}} \log \theta_{2}\left(k z, q^{k}\right)\right|_{z=0} \\
& =-8 q \frac{d}{d q} \log \left(\frac{\eta(2 k \tau)^{2}}{\eta(k \tau)}\right)+k^{2}\left(-8 q^{k} \frac{d}{d q^{k}} \log \left(\frac{\eta(k \tau)}{\eta(2 k \tau)^{2}}\right)\right) \\
& =8(k-1) q \frac{d}{d q} \log \left(\frac{\eta(2 k \tau)^{2}}{\eta(k \tau)}\right),
\end{aligned}
$$

and application of $(2.5)$ and 2.7$)$ imply that

$$
\begin{aligned}
& \left.\mathrm{T}_{z, q}\left(\log \left(\frac{\theta_{2}\left(k z-\pi / 2, q^{k}\right)}{\theta_{2}(z-\pi / 2, q)}\right)\right)\right|_{z=0} \\
= & -8 q \frac{d}{d q} \log \left(\frac{\eta(k \tau)^{3}}{\eta(\tau)^{3}}\right)-\left.\frac{\partial^{2}}{\partial z^{2}} \log \left(\frac{\theta_{2}\left(k z-\pi / 2, q^{k}\right)}{\theta_{2}(z-\pi / 2, q)}\right)\right|_{z=0} \\
= & -24 q \frac{d}{d q} \log \left(\frac{\eta(k \tau)}{\eta(\tau)}\right)-8 q \frac{d}{d q} \log \left(\frac{\eta(\tau)}{\eta(k \tau)^{k}}\right) \\
= & 8 q \frac{d}{d q} \log \left(\eta(k \tau)^{k-3} \eta(\tau)^{2}\right),
\end{aligned}
$$

which completes the proof of the lemma.

We need the following half product formula for Jacobi theta function $\theta_{2}$, which will be used to prove Theorem 1.4 in the next section.

Lemma 2.4. For integer $k \geq 1$ and $\delta \in\{0,1\}$,

$$
\prod_{\substack{0 \leq \ell<2 k \\ \ell-k \equiv \delta(\bmod 2)}} \theta_{2}\left(z+\frac{\ell}{2 k} \pi, q\right)=C_{k, \delta} \frac{\eta(\tau)^{k}}{\eta(k \tau)} \theta_{2}\left(k z+\frac{(\delta-1) \pi}{2}, q^{k}\right),
$$

where $C_{k, \delta}=e^{\frac{\mathbf{i} \pi}{2}\left(\delta-k+\mathbf{1}_{k \neq \delta(\bmod 2)}\right)}$. Here and throughout, $\mathbf{1}_{\text {condition }}=1$ if the 'condition' is true, and equals to 0 if the 'condition' is false. 
Proof. From 2.1 we have

$$
\begin{aligned}
\prod_{\substack{0 \leq \ell<2 k \\
\ell-k \equiv \delta(\bmod 2)}} \theta_{2}\left(z+\frac{\ell \pi}{2 k}, q\right)= & \prod_{\substack{0 \leq \ell<2 k \\
\ell-k \equiv \delta(\bmod 2)}}\left(q^{1 / 8} e^{-\mathrm{i}\left(z+\frac{\ell}{2 k} \pi\right)} \prod_{n \geq 1}\left(1-q^{n}\right)\right) \\
& \times \prod_{\substack { n \geq 1 \\
\begin{subarray}{c}{\ell \leq k \equiv \delta(\bmod 2){ n \geq 1 \\
\begin{subarray} { c } { \ell \leq k \equiv \delta ( \operatorname { m o d } 2 ) } }\end{subarray}}\left(1+q^{n} e^{-2 \mathrm{i} z-\frac{\ell \pi \mathrm{i}}{k}}\right)\left(1+q^{n-1} e^{2 \mathrm{i} z+\frac{\ell \pi \mathrm{i}}{k}}\right) .
\end{aligned}
$$

It is easy to check that

$$
\prod_{\substack{0 \leq \ell<2 k \\ \ell-k \equiv \delta(\bmod 2)}}\left(1+x e^{ \pm \ell \pi \mathrm{i} / k}\right)=1-e^{\delta \pi \mathrm{i}} x^{k} \quad \text { and } \sum_{\substack{0 \leq \ell<2 k \\ \ell-k \equiv \delta(\bmod 2)}} \ell=k(k-1)+k \mathbf{1}_{k \neq \delta(\bmod 2)} .
$$

Thus we obtain that

$$
\begin{aligned}
\prod_{\substack{0 \leq \ell<2 k \\
\ell-k \equiv \delta(\bmod 2)}} \theta_{2}\left(z+\frac{\ell}{2 k} \pi, q\right)= & q^{k / 12} \eta(\tau)^{k} e^{-\mathrm{i} k z} e^{-\frac{\mathrm{i} \pi}{2}\left(k-1+\mathbf{1}_{k \neq \delta(\bmod 2)}\right)} \\
& \times \prod_{n \geq 1}\left(1-e^{-2 \mathrm{i} k z-\delta \pi \mathrm{i}} q^{k n}\right)\left(1-e^{2 \mathrm{i} k z+\delta \pi \mathrm{i}} q^{k(n-1)}\right) \\
= & C_{k, \delta} \theta_{2}\left(k z+(\delta-1) \pi / 2, q^{k}\right) \frac{\eta(\tau)^{k}}{\eta(k \tau)}
\end{aligned}
$$

with

$$
C_{k, \delta}=e^{\frac{\mathrm{i} \pi(\delta-1)}{2}-\frac{\mathrm{i} \pi\left(k-1+\mathbf{1}_{k \neq \equiv \delta(\bmod 2)}\right)}{2}}=e^{\frac{\mathrm{i} \pi}{2}\left(\delta-k+\mathbf{1}_{k \neq \equiv(\bmod 2)}\right)},
$$

which completes the proof of the lemma.

$$
\text { 3. Proof of Theorem } 1.4
$$

First of all, we define

$$
G_{\delta, k}(z, q):=\sum_{\substack{0 \leq \ell<k \\ \ell-k \equiv \delta(\bmod 2)}}\left[\frac{\partial}{\partial z} \log \theta_{2}\left(z+\frac{\ell}{2 k} \pi, q\right)\right]^{2}
$$

then from 1.4 we have $S_{\delta}(k)=G_{\delta, k}(0, q)$. By Proposition 2.1 we get

$$
\begin{aligned}
G_{\delta, k}(z, q) & =\sum_{\substack{0 \leq \ell<k \\
\ell-k \equiv \delta(\bmod 2)}} \mathrm{T}_{z, q}\left(\log \theta_{2}\left(z+\frac{\ell}{2 k} \pi, q\right)\right) \\
& =\mathrm{T}_{z, q}\left(\sum_{\substack{0 \leq \ell<k \\
\ell-k \equiv \delta(\bmod 2)}} \log \theta_{2}\left(z+\frac{\ell}{2 k} \pi, q\right)\right) .
\end{aligned}
$$


We shall define the auxiliary function as follows:

$$
A_{\delta, k}(z, q)=\mathrm{T}_{z, q}\left(\sum_{\substack{0 \leq \ell \leq 2 k \\ \ell-k \equiv \delta(\bmod 2)}} \log \theta_{2}\left(z+\frac{\ell \pi}{2 k}, q\right)-\mathbf{1}_{\delta=0} \log \theta_{2}\left(z+\frac{\pi}{2}, q\right)\right) .
$$

It is clear that $A_{\delta, k}(0, q):=\lim _{z \rightarrow 0} A_{\delta, k}(z, q)$ exists. We claim that

$$
G_{\delta, k}(0, q)=\frac{1}{2} A_{\delta, k}(0, q) .
$$

In fact, by $\theta_{2}(z+\pi, q)=-\theta_{2}(z, q)$ and $\theta_{2}(z, q)=\theta_{2}(-z, q)$ we have

$$
\begin{aligned}
A_{\delta, k}(z, q) & =\sum_{\substack{0 \leq \ell<k \\
\ell-k \equiv \delta(\bmod 2)}} \mathrm{T}_{z, q}\left(\log \theta_{2}\left(z+\frac{\ell \pi}{2 k}, q\right)+\log \theta_{2}\left(z+\frac{(2 k-\ell) \pi}{2 k}, q\right)\right) \\
& =\sum_{\substack{0 \leq \ell<k \\
\ell-k \equiv \delta(\bmod 2)}} \mathrm{T}_{z, q}\left(\log \theta_{2}\left(z+\frac{\ell}{2 k} \pi, q\right)+\log \left(-\theta_{2}\left(-z+\frac{\ell}{2 k} \pi, q\right)\right)\right) .
\end{aligned}
$$

Further, by the definition of $\mathrm{T}_{z, q}$, we see that

$$
\begin{aligned}
& \left.\mathrm{T}_{z, q}\left(\log \left(-\theta_{2}\left(-z+\frac{\ell}{2 k} \pi, q\right)\right)\right)\right|_{z=0} \\
= & -8 q \frac{\partial}{\partial q}\left(\log \theta_{2}\left(\frac{\ell}{2 k} \pi, q\right)\right)-\left.\frac{\partial^{2}}{\partial z^{2}}\left(\log \theta_{2}\left(-z+\frac{\ell}{2 k} \pi, q\right)\right)\right|_{z=0} \\
= & -8 q \frac{\partial}{\partial q}\left(\log \theta_{2}\left(\frac{\ell}{2 k} \pi, q\right)\right)-\left.\frac{\partial^{2}}{\partial z^{2}}\left(\log \theta_{2}\left(z+\frac{\ell}{2 k} \pi, q\right)\right)\right|_{z=0} \\
= & \left.\mathrm{T}_{z, q}\left(\log \theta_{2}\left(z+\frac{\ell}{2 k} \pi, q\right)\right)\right|_{z=0},
\end{aligned}
$$

which immediately obtain the proof of (3.1).

On the other hand, from Lemma 2.4 we find that

$$
\begin{aligned}
\sum_{\substack{0 \leq \ell<2 k \\
\ell-k \equiv \delta(\bmod 2)}} \log \theta_{2}\left(z+\frac{\ell \pi}{2 k}, q\right) & =\log \prod_{\substack{0 \leq \ell<2 k \\
\ell-k \equiv \delta(\bmod 2)}} \theta_{2}\left(z+\frac{\ell}{2 k} \pi, q\right) \\
& =\log \left(C_{k, \delta} \frac{\eta(\tau)^{k}}{\eta(k \tau)} \theta_{2}\left(k z+\frac{(\delta-1) \pi}{2}, q^{k}\right)\right),
\end{aligned}
$$

which implies that

$$
\begin{aligned}
A_{\delta, k}(z, q)= & \mathrm{T}_{z, q}\left(\log \theta_{2}\left(k z+\frac{(\delta-1) \pi}{2}, q^{k}\right)+\log \left(C_{k, \delta} \frac{\eta(\tau)^{k}}{\eta(k \tau)}\right)\right) \\
& +\mathrm{T}_{z, q}\left(\mathbf{1}_{k \equiv \delta(\bmod 2)} \log \theta_{2}(z, q)\right)-\mathrm{T}_{z, q}\left(\mathbf{1}_{\delta=0} \log \theta_{2}\left(z+\frac{\pi}{2}, q\right)\right)
\end{aligned}
$$


Further by Lemma 2.3 we obtain that

$$
\begin{aligned}
A_{0, k}(0, q)= & \left.\mathrm{T}_{z, q}\left(\log \left(\frac{\theta_{2}\left(k z-\pi / 2, q^{k}\right)}{\theta_{2}(z-\pi / 2, q)}\right)\right)\right|_{z=0} \\
& -8 q \frac{\partial}{\partial q} \log \left(\frac{\eta(\tau)^{k}}{\eta(k \tau)}\right)+\left.\mathbf{1}_{k \equiv 0(\bmod 2)} \mathrm{T}_{z, q}\left(\log \theta_{2}(z, q)\right)\right|_{z=0} \\
= & 8 q \frac{d}{d q} \log \left(\eta(k \tau)^{k-3} \eta(\tau)^{2}\right)-8 q \frac{d}{d q} \log \left(\frac{\eta(\tau)^{k}}{\eta(k \tau)}\right) \\
= & 8(k-2) q \frac{d}{d q} \log \left(\frac{\eta(k \tau)}{\eta(\tau)}\right)
\end{aligned}
$$

and

$$
\begin{aligned}
A_{1, k}(0, q)= & \left.\mathrm{T}_{z, q}\left(\log \theta_{2}\left(k z, q^{k}\right)\right)\right|_{z=0} \\
& -8 q \frac{\partial}{\partial q} \log \left(\frac{\eta(\tau)^{k}}{\eta(k \tau)}\right)+\left.\mathbf{1}_{k \equiv 1(\bmod 2)} \mathrm{T}_{z, q}\left(\log \theta_{2}(z, q)\right)\right|_{z=0} \\
= & 8(k-1) q \frac{d}{d q} \log \left(\frac{\eta(2 k \tau)^{2}}{\eta(k \tau)}\right)-8 q \frac{d}{d q} \log \left(\frac{\eta(\tau)^{k}}{\eta(k \tau)}\right) \\
= & 8 q \frac{d}{d q} \log \left(\frac{\eta(2 k \tau)^{2 k-2}}{\eta(\tau)^{k} \eta(k \tau)^{k-2}}\right)
\end{aligned}
$$

which completes the proof of Theorem 1.4 by noting that $S_{\delta}(k)=G_{\delta, k}(0, q)=\frac{1}{2} A_{\delta, k}(0, q)$.

\section{Acknowledgments}

The author would like to thank the anonymous referees for their very helpful comments and suggestions. The author also thank his advisor Zhi-Guo Liu for consistent encouragement and useful suggestions.

\section{References}

[1] G. E. Andrews, A simple proof of Jacobi's triple product identity, Proc. Amer. Math. Soc. 16 (1965), 333-334.

[2] H. M. Farkas and I. Kra, Theta Constants, Riemann Surfaces and the Modular Group: An introduction with applications to uniformization theorems, partition identities and combinatorial number theory, Graduate Studies in Mathematics 37, American Mathematical Society, Providence, RI, 2001.

[3] _ On theta constant identities and the evaluation of trigonometric sums, in: Complex Manifolds and Hyperbolic Geometry (Guanajuato, 2001), 115-131, Contemp. Math. 311, Amer. Math. Soc., Providence, RI, 2002. 
[4] E. T. Whittaker and G. N. Watson, A Course of Modern Analysis: An introduction to the general theory of infinite processes and of analytic functions: with an account of the principal transcendental functions, Fourth edition, Cambridge University Press, New York, 1962.

Nian Hong Zhou

School of Mathematical Sciences, East China Normal University, 500 Dongchuan Road, Shanghai 200241, P. R. China

E-mail address: nianhongzhou@outlook.com 